\begin{tabular}{|c|l|}
\hline Title & HTS split open-ring resonators with improved power-handling capability for reaction-type transmitting filters \\
\hline Author(s) & Futatsumori, S.; Hikage, T.; Nojima, T.; A kasegawa, A.; Nakanishi, T.; Y amanaka, K. \\
\hline Citation & $\begin{array}{l}\text { Electronics Letters, 43(17), 956-957 } \\
\text { https:/doi.org/40.1049/1/20071684 }\end{array}$ \\
\hline Issue Date & 2007-08-16 \\
\hline Doc URL & http://hdl.handle.net/2115/30156 \\
\hline Rights & $\begin{array}{l}\text { This paper is a postprint of a paper submitted to and accepted for publication in ELECTRONICS LETTERS and is } \\
\text { subject to Institution of Engineering and Technology Copyright. The copy of record is avail lable at IET Digital Library. }\end{array}$ \\
\hline Type & article (author version) \\
\hline File Information & EL43-17.pdf \\
\hline
\end{tabular}

Instructions for use 


\title{
HTS split open-ring resonators with improved power-handling capability for reaction-type transmitting filters
}

\author{
S. Futatsumori, T. Hikage, T. Nojima, A. Akasegawa, T. Nakanishi, and K. \\ Yamanaka
}

\begin{abstract}
Novel planar construction of high-temperature superconducting filters that improve power-handling capability is proposed. Split open-ring resonators are developed to be used in reaction-type transmitting filters that have very sharp bandstop characteristics. Resonator structures to achieve high-unloaded $Q$-factors and low radiation as well as low surface current densities in the passband are numerically investigated for $5 \mathrm{GHz}$ band operation. Low surface current densities reduce the generation of intermodulation distortions. Measurements confirm an unloaded $Q$-factor of 68000 and third intermodulation-distortion of -82 $\mathrm{dBc}$ at $30 \mathrm{dBm}$ input power.
\end{abstract}

Introduction: Planar microwave filters based on high-temperature superconducting (HTS) thin-films achieve simultaneous steep skirt characteristics, low insertion loss, and small volume at the same time, unlike conventional room-temperature devices [1]. However, HTS microwave filters for high-power applications that have the same performance as HTS receiving filters have not been realised so far. This is mainly due to surface-current concentrations on the devices and the generation of intermodulation distortion [2, 3]. We proposed the HTS reaction-type transmitting filter (HTS-RTF) to suppress adjacent noise generated by the power amplifier [4]. Since reaction-type resonators do not resonate with high-power fundamental signals, an HTS-RTF offers both high-power handling capability and sharp cutoff characteristics. For practical applications in the microwave region, however, the resonators must 
achieve low surface current densities in the passband and high-unloaded $Q$-factors as well as low radiation levels.

This Letter proposes a novel HTS split open-ring resonator (SORR) with improved power-handling capability for HTS-RTFs. First, novel SORR structures that reduce maximum current densities while offering both high-unloaded $Q$-factors and low radiations are investigated in the $5 \mathrm{GHz}$ band. Secondly, to further improve the current density dispersion effect, additional slits are added to the resonator surface. Finally, the characteristics of the proposed SORR with slits are investigated experimentally. To confirm the effects of the SORR with slits, resonator unloaded $Q$-factor and third intermodulation-distortion (IMD3) are compared with those of the conventional open-ring resonator.

Split open-ring resonator: Since HTS-RTFs have the bandstop characteristic, their resonators do not, ideally, resonate at passband frequencies. However, as the stopband and passband edges are very close to each other, passband signals are able to induce currents on the resonators. These induced currents have the possibility of exceeding HTS critical current density or generate intermodulation distortion. To reduce these induced current densities, a $2 \mathrm{GHz}$ SORR was proposed and the edge-current dispersion effect was numerically investigated in a previous letter [4]. Additional investigations are carried out to improve resonator characteristics in the 5 $\mathrm{GHz}$ band. The investigation is based upon full wave electromagnetic analysis using the method of moments. The geometry of a $5 \mathrm{GHz}$ SORR is shown in Fig. 1a. The material of the substrate is assumed to be $\mathrm{MgO}$ with dielectric constant of 9.7 , a loss tangent of $5.5 \times 10^{-6}$, and a thickness of $0.5 \mathrm{~mm}$. The HTS thin-film material is assumed to be YBCO with conductivity of $6.5 \times 10^{12} \mathrm{~S} / \mathrm{m}$ and thickness of $0.5 \mathrm{um}$. In addition, a shielded box with finite conductivity the top plate height of which is $5 \mathrm{~mm}$ is taken into account.

Fig. 2 shows maximum induced current density against resonator gap $g$. The maximum induced current density of an SORR is decreased by $27.2 \%$ at $g=0.4 \mathrm{~mm}$ 
compared to the open-ring resonator shown in Fig. 1b. Furthermore, unloaded $Q$-factor of the SORR is 47800 at $g=0.4 \mathrm{~mm}$. For both resonators, diameters and distance of resonators to feedlines are adjusted to achieve the coupling coefficient $\beta_{\mathrm{e}}$ $=10$ and to resonate at $4.950 \mathrm{GHz}$. In addition, assuming that the fundamental signal lies in the passband, input signal is a sinusoidal wave with $1 \mathrm{~V}$ amplitude and $5 \mathrm{MHz}$ offset from resonance frequency. The radiation energy from the resonators is estimated. The total unloaded quality factor of the resonator can be found by adding loss of the conductor, dielectric substrate, and radiation, as follows:

$$
\frac{1}{Q_{u}}=\frac{1}{Q_{c}}+\frac{1}{Q_{d}}+\frac{1}{Q_{r}}
$$

where $Q_{c}, Q_{d}$ and $Q_{r}$ are the conductor, dielectric, and radiation quality factors. To avoid cross coupling and degradation of the filter characteristics, radiation should be suppressed as much as possible. Fig. 3 shows radiation quality factor $Q_{r}$ against resonator gap $g$, as calculated from the frequency characteristics without a shielding box. Since the current directions on the two elements of the SORR are opposite, the resonator has a radiation cancelling effect. Narrower gaps lead to lower radiation energy. These results indicate that the optimal resonator gap $g=0.3 \mathrm{~mm}$ since it enables both low current density and radiation energy. The SORR with $g=0.3 \mathrm{~mm}$ achieves radiation quality factor $Q_{r}$ of 32000 which is more than twice that of the open-ring resonator (15 100), as well as having a current density dispersion effect of $23.2 \%$ and unloaded $Q$-factor of 48100 .

Split open-ring resonator with slits: We propose here a current density dispersion method that further suppresses the edge-current concentration. Fig. 4a shows the open-ring resonator with slits. The 20 um wide slits are aligned to the direction of current flow and not degrade overall performance. Fig. 5 shows the maximum induced current density versus the number of slits. As the number of slits is increased, maximum induced current density is reduced. To prevent the unwanted resonance mode, it is important to connect the end of the resonator. Since the width of the 
resonator is fixed, increasing the number of slits leads to a reduction in the conducting area and low unloaded $Q$-factors. Note the reduction in the current density dispersion effect when the number of slits is seven. Our solution is to add three slits to the SORR with $g=0.3 \mathrm{~mm}$, as shown in Fig. $4 \mathrm{~b}$. The current density reduction effect is improved to $37.1 \%$, from the $23.2 \%$ of the SORR without slits.

Fabrication and measurements: To validate the analysis results, the SORR with three slits (Fig. 4b) and the open-ring resonator (Fig. 1a) were fabricated and measured. Note that the coupling coefficient and resonant frequency are adjusted to the values described above. The measured unloaded $Q$-factor of the SORR with three slits and the open-ring resonator are 68,000 and 64,300 at $50 \mathrm{~K}$, respectively. Nonlinear distortion measurements using a 2-tone signal were then carried out. Assuming the passband signals, input signals are separated by $500 \mathrm{kHz}$ and are $5 \mathrm{MHz}$ offset from the resonance frequency. Fig. 6 shows the measured IMD3 of the two resonators at $50 \mathrm{~K}$. IMD3 of the SORR with three slits is $-82.2 \mathrm{dBc}$ at $30 \mathrm{dBm}$ input power, which is about $13 \mathrm{~dB}$ lower than that of the open-ring resonator. This is about a $4 \mathrm{~dB}$ improvement in terms of input power. The SORR with slits reduces the maximum current density while keeping the $Q$-factor high, which confirms the effectiveness of the proposed structure.

Conclusions: SORRs with improved power-handling capability have been proposed. The SORRs with three slits, which reduce the maximum current density in the passband while achieving both high-unloaded $Q$-factors and low radiation, were designed based on a numerical analysis. The measured results confirm the effectiveness of the proposed structure.

Acknowledgments: This work is partly supported by Grant-in-Aid for Research \& Development for Efficient Use of Frequency Spectrum Resources from Ministry of Internal Affairs and Communications (MIC) of Japan. 


\section{References}

[1] HONG, J. -S., McErlean, E. P., and Karyamapudi, B.: 'Narrowband high temperature superconducting filter for mobile communication systems'. IEE Proc.-Microw. Antennas Propag., 2004, 151, (6), pp.491-496

[2] SHEN, Z. -Y., WILKER, C., PANG, P., FACE, D. W., CARTER III, C. F., and HARRINGTON, C. M.: 'Power handling capability improvement of High-Temperature Superconducting microwave circuits'. IEEE Trans. Applied Supercond., 1997, 7, (2), pp.2446-2453

[3] SATO, S., KURIAN, J., and NAITO, M.: 'Third-order intermodulation measurements of microstrip Bandpass filter based on High-Temperature Superconductors'. IEEE Trans. Microwave Theory Tech., 2004, 52, (12), pp.2658-2663

[4] FUTATSUMORI, S., HIKAGE, T., and NOJIMA, T.: 'Microwave superconducting reaction-type transmitting filter using split open-ring resonator'. Electoron. Lett., 2006, 42, (7), pp.428-430

Author's affiliations:

S. Futatsumori, T. Hikage, and T. Nojima ( Graduate School of Information Science and Technology, Hokkaido University, Kita 14, Nishi 9, Kita-ku, Sapporo, Hokkaido, 060-0814, Japan )

A. Akasegawa, T. Nakanishi, and K. Yamanaka ( Fujitsu Limited, 10-1 Morinosato-Wakamiya, Atsugi, Kanagawa, 243-0197 Japan )

Corresponding author :

S. Futatsumori ( E-mail : futatsumori @ emwtinfo.ice.eng.hokudai.ac.jp ) 
Figure and table captions:

Fig. 1 Structures of split open-ring resonator and open-ring resonator (all dimensions in millimetres)

a Split open-ring resonator

b Open-ring resonator

Fig. 2 Maximum induced current density and unloaded Q-factor against resonator gap $g$

ㄴ- maximum induced current density

$\multimap \quad$ unloaded $Q$-factor

Fig. 3 Radiation quality factor $Q_{r}$ against resonator gap $g$

Fig. 4 Structure of open-ring resonator with slits and split open-ring resonator with slits (all dimensions in millimetres)

a Open-ring resonator with slits

b Split open-ring resonator with slits

Fig. 5 Number of slits against maximum induced current density

Fig. 6 Measured third intermodulation distortion values

-

$\longrightarrow$ - third intermodulation-distortion of open-ring resonator

$\triangle \quad$ third intermodulation-distortion of split open-ring resonator with slits 
Figure 1
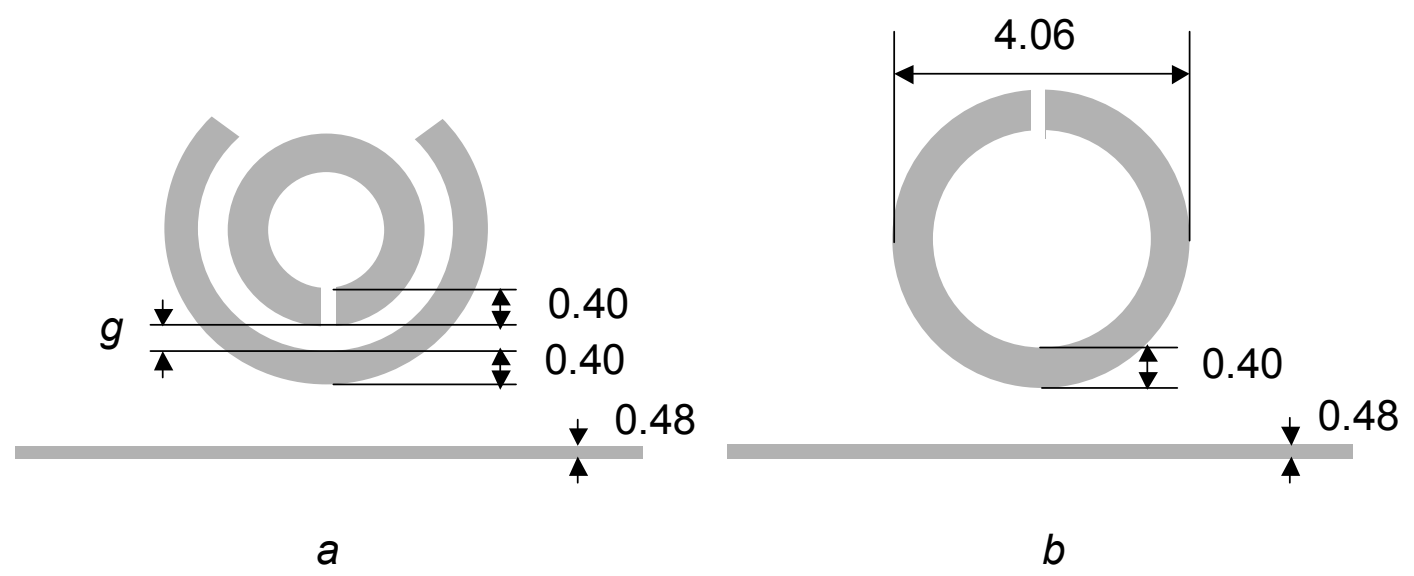
Figure 2

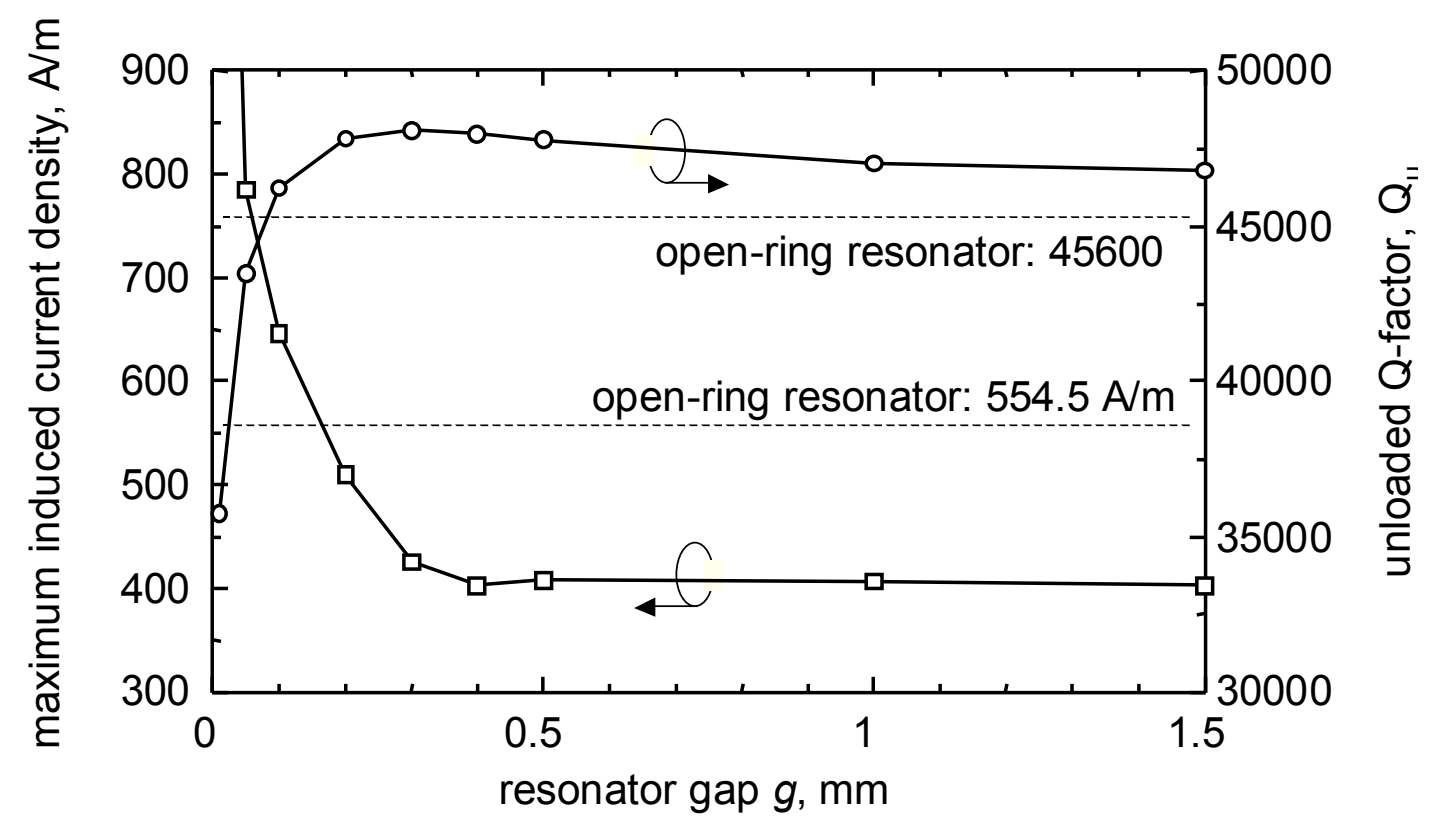


Figure 3

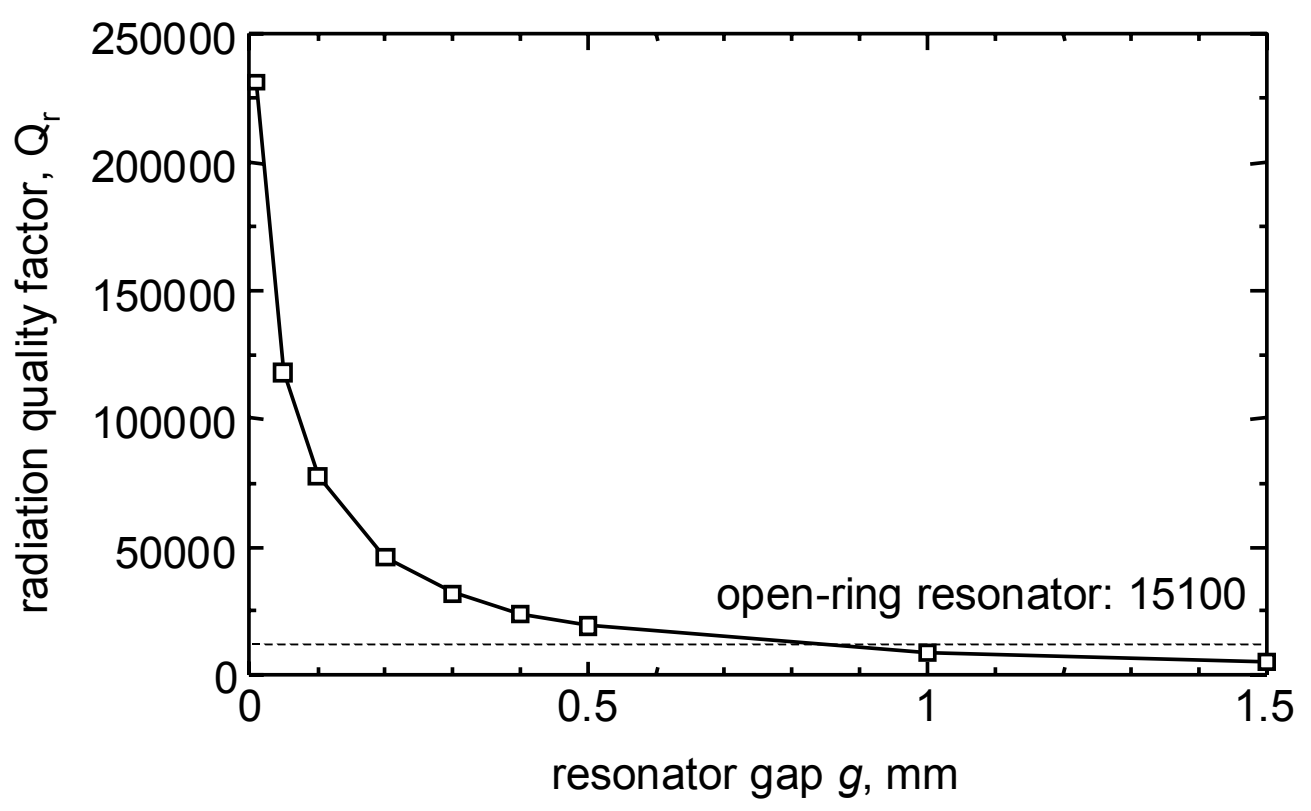


Figure 4

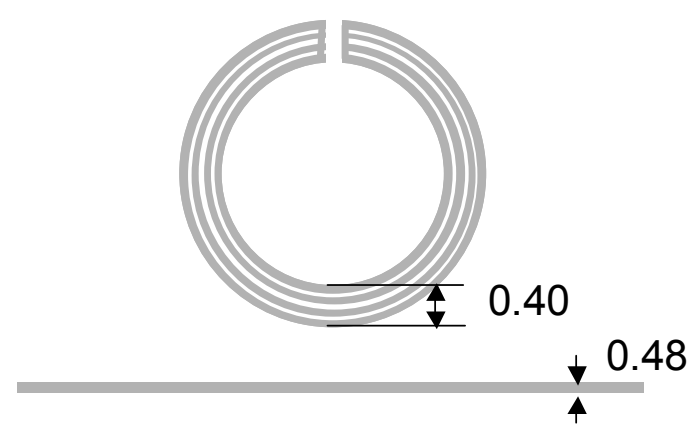

a

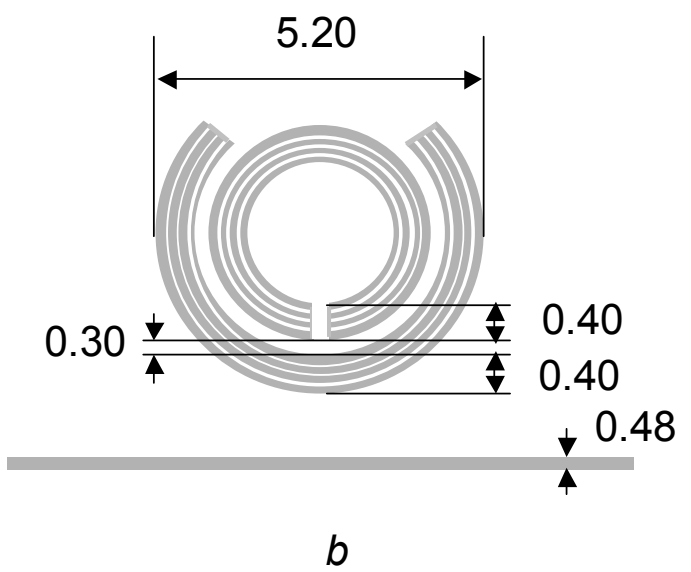


Figure 5

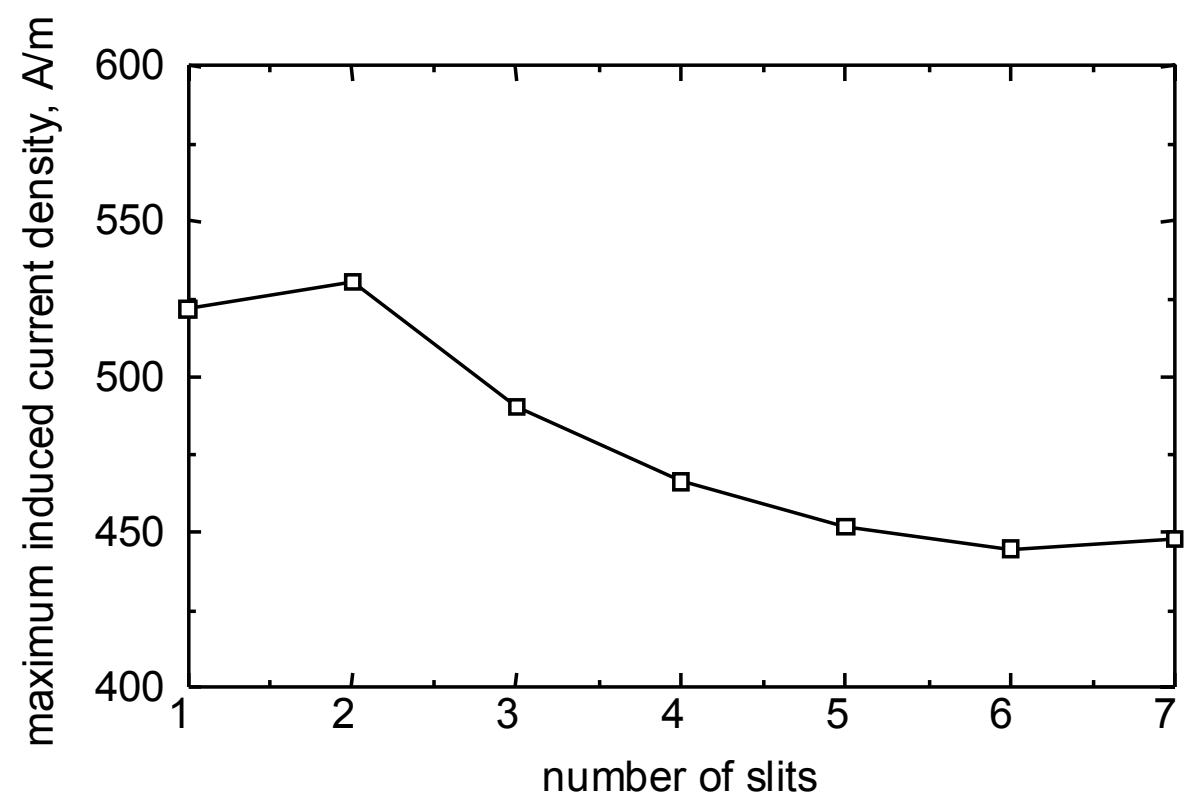


Figure 6

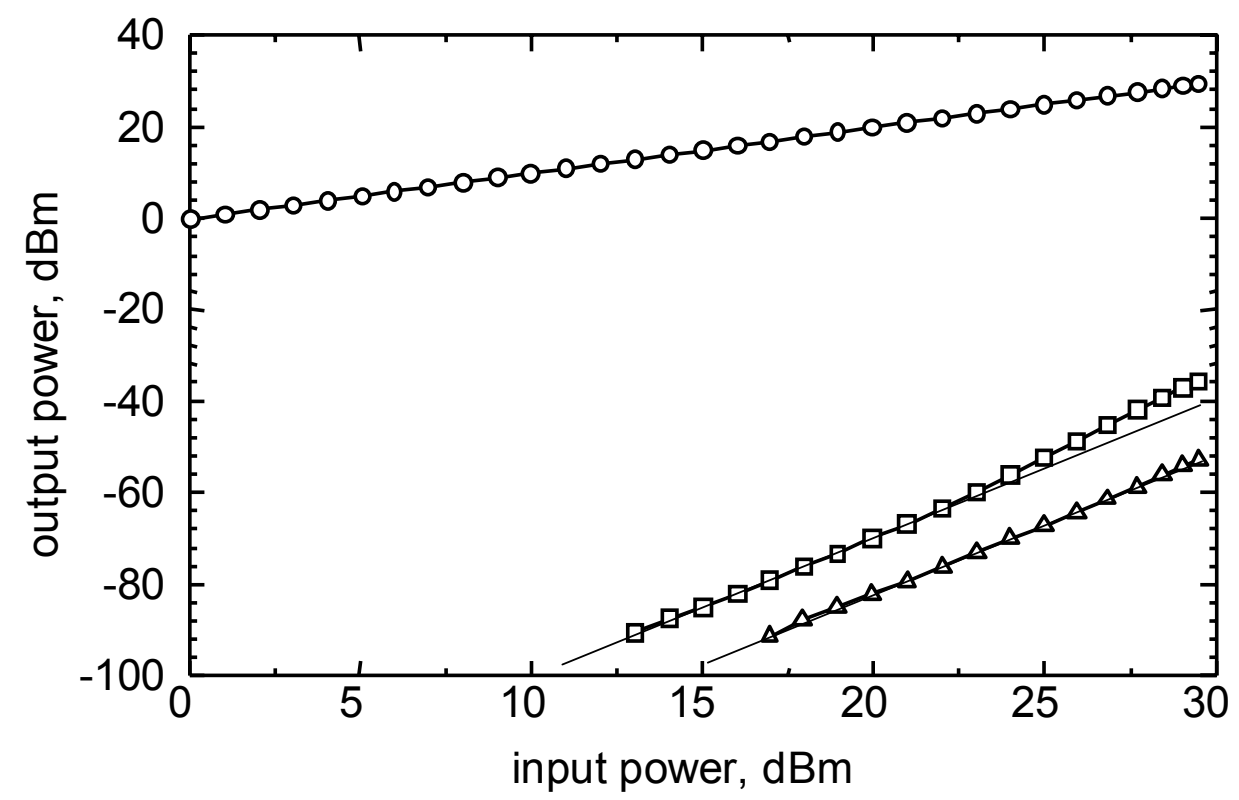

